\title{
On the Bifurcations of Dean Flow between Porous Horizontal Cylinders with a Radial Flow and a Radial Temperature Gradient
}

\author{
Luiza Shapakidze \\ A. Razmadze Mathematical Institute of I. Javakhishvili Tbilisi State University, Tbilisi, Georgia \\ Email: luiza.shapakidze@tsu.ge
}

How to cite this paper: Shapakidze, L. (2017) On the Bifurcations of Dean Flow between Porous Horizontal Cylinders with a Radial Flow and a Radial Temperature Gradient. Journal of Applied Mathematics and Physics, 5, 1725-1738.

https://doi.org/10.4236/jamp.2017.59146

Received: August 7, 2017

Accepted: September 16, 2017

Published: September 19, 2017

Copyright $\odot 2017$ by author and Scientific Research Publishing Inc. This work is licensed under the Creative Commons Attribution International License (CC BY 4.0). http://creativecommons.org/licenses/by/4.0/

\begin{abstract}
The stability of a heat-conducting flow due to the pumping of a fluid around the annulus of horizontal porous cylinders is studied. The basic flow is under the action of radial flow and a radial temperature gradient. The objects of investigations are different regimes and bifurcations which may arise in this flow.
\end{abstract}

\section{Keywords}

Dean Flow, Porous Cylinders, Transitions, Instability, Bifurcations

\section{Introduction}

Due to the technological and engineering importance, the investigation of the stability of curved flows has been a source of interest for several years.

Experimental studies of main flow in curved pipes carried out by Eustice [1] [2] pointed out the secondary flow formation that analytically confirmed by Dean [3]. His stability analysis has shown that flow between two horizontal cylinders becomes unstable for small axisymmetric disturbances when the Dean number exceeds the critical value. Above this value, there appear pairs of counter-rotating streamwise-oriented vortices known as Dean vortex flows. Later on, this theoretical analysis has been confirmed by W. Reid [4], G. Hammerlin [5] and experimentally by D. Browster et al. [6].

The stability investigation of Dean flow was continued by many authors (see [7]-[13]).

Important results were obtained also in the study of Dean flow stability of complex fluids. [14] and [15] present experimental and numerical analysis of the 
behavior of physiological flow in a curved pipe. The numerical study of magnetohydrodynamic viscous steady biofluid flow through a curved pipe with circular cross section can be found in [16]. [17] is devoted to the analytical study of conductive fluids in highly conductive curved pipes. The influence of porous channel with a radial flow and the effect of a radial temperature gradient on the stability of Dean flow have been considered in [18] [19]. The Dean flow stability of a heat-conducting fluid between horizontal porous cylinders with a radial temperature gradient and a radial flow has been studied in [20] [21].

In these papers, investigations are mainly carried out in the linear approximation, which allows one to judge the first loss of stability of main flow. It was shown that the first instability of the basic steady flow can be not only a monotonic instability leading to the Dean vortices but also can be oscillatory instability resulting in a time-periodic secondary regime.

For practical purposes, it is important to consider the transitions to more complex regimes, the appearance of which precedes the development of higher instability and leads to turbulence.

For Dean flow stability, the numerical and experimental investigations of transitions to turbulence were carried out, for instance, in [22] [23] [24] [25]. In [22], the linear stability of axisymmetric Dean vortex flow to non-axisymmetric perturbations is considered. Two different nonaxisymmetric flows, undulating and twisting Dean vortex flow, are found to be associated with the two transitions. In [23], the stability of the Dean-type flow is studied experimentally and comparied with instabilities and transitions in the Taylor-Couette flow. In [24], the effect of curvature on laminar channel flow is studied experimentally. It is shown that two types of secondary instabilities, with distinctly different frequencies, are at hand simultaneously, and their spatial distribution and growth are determined. In [25], numerical study of transitions that occur with increasing Reynolds number in a curved chanel is performed.

Our aim is to study possible transitions to complex modes in a heat-coducting flow between horizontal porous cylinders with radial flow and radial temperature gradient. The linear analysis of that problem for small axisymmetric perturbations has been performed in [20] [21]. Investigating the transitions to more complex modes, we are interested in the structures of transitions to turbulence in this flow, depending on the parameters of the problem. Based on the nonlinear analysis, we can predict the existence of quasiperiodic oscillations and chaotic regimes for certain small absolute values of parameters of the problem.

\section{Formulation of the Problem}

We consider the steady flow in an annulus between the fixed horizontal heated cylinders to be maintained by a constant azimuthal pressure gradient in the presence of a radial flow through the porous cylinders and of a radial temperature gradient. We denote the radii and temperature of the inner and outer cylinders by $R_{1}, R_{2}$ and $T_{1}, T_{2}$, respectively. A constant flow through the annulus is 


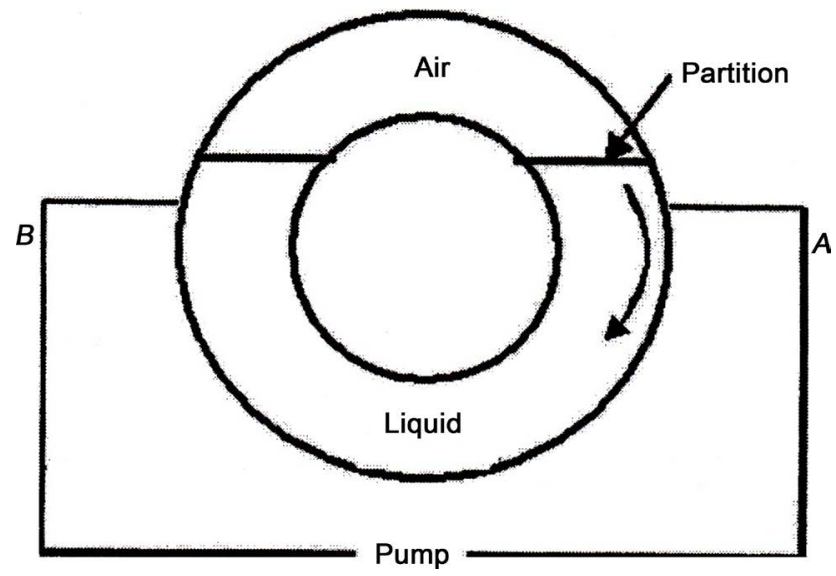

Figure 1. Horizontal concentric cylinder system.

maintained by a suitable pumping circuit. It is assumed that the fluid inflow through one cylinder is equal to the fluid outflow through the other. The system that will be considered here is shown in Figure 1. Point $A$ denotes the delivery side, while $B$ denotes the suction side of the pump. The annulus is partially filled with the liquid. There is a partition in the annulus at the air-liquid interface to maintain the azimuthal pressure gradient. The radial flow through the porous cylinders may be either outward or inward.

In the cylindrical coordinates $(r, \varphi, z)$ with the $z$-axis coinciding with that of the cylinders, the Navier-Stokes equations as wall as those for heat transfer, continuity and state are the form [26]:

$$
\begin{aligned}
& \frac{\partial v_{r}^{\prime}}{\partial t}+\left(V^{\prime}, \nabla\right) v_{r}^{\prime}-\frac{v_{\varphi}^{\prime 2}}{r}+\frac{1}{\rho^{\prime}} \frac{\partial \Pi^{\prime}}{\partial r}=v\left(\Delta v_{r}^{\prime}-\frac{v_{r}^{\prime}}{r^{2}}-\frac{2}{r^{2}} \frac{\partial v_{\varphi}^{\prime}}{\partial \varphi}\right), \\
& \frac{\partial v_{\varphi}^{\prime}}{\partial t}+\left(V^{\prime}, \nabla\right) v_{\varphi}^{\prime}+\frac{v_{\varphi}^{\prime} v_{r}^{\prime}}{r}+\frac{1}{r \rho^{\prime}} \frac{\partial \Pi^{\prime}}{\partial \varphi}=v\left(\Delta v_{\varphi}^{\prime}-\frac{v_{\varphi}^{\prime}}{r^{2}}+\frac{2}{r^{2}} \frac{\partial v_{r}^{\prime}}{\partial \varphi}\right), \\
& \frac{\partial v_{z}^{\prime}}{\partial t}+\left(V^{\prime}, \nabla\right) v_{z^{\prime}}+\frac{1}{\rho^{\prime}} \frac{\partial \Pi^{\prime}}{\partial z}=v \Delta v_{z}^{\prime}, \quad \frac{\partial T^{\prime}}{\partial t}+\left(V^{\prime}, \nabla\right) T^{\prime}=\chi \Delta T^{\prime}, \\
& \frac{\partial \rho^{\prime}}{\partial t}+\operatorname{div}\left(\rho^{\prime} V^{\prime}\right)=0, \quad \rho^{\prime}=\rho_{0}\left(1-\beta\left(T^{\prime}-T_{0}\right)\right),
\end{aligned}
$$

where $V^{\prime}=\left\{v_{r}^{\prime}, v_{\varphi}^{\prime}, v_{z}^{\prime}\right\}$ is the velocity vector; $T^{\prime}$ is temperature; $\Pi^{\prime}$ is pressure; $\rho^{\prime}$ is the fluid density; $t$ is time; $v, \chi, \beta$ are, respectively, the coefficients of kinematic viscosity, thermal diffusivity and thermal expansion, $\rho_{0}$; and $T_{0}$ denote constant density and temperature, respectively.

Operators $\Delta$ and $\nabla$ in the cylindrical coordinates are of the form

$$
\Delta=\frac{\partial^{2}}{\partial r^{2}}+\frac{1}{r} \frac{\partial}{\partial r}+\frac{1}{r^{2}} \frac{\partial^{2}}{\partial \varphi^{2}}+\frac{\partial^{2}}{\partial z^{2}}, \quad \nabla=\left\{\frac{\partial}{\partial r}, \frac{1}{r} \frac{\partial}{\partial \varphi}, \frac{\partial}{\partial z}\right\} .
$$

Equation (1) are considered under the condition

$$
\int_{0}^{2 \pi} \int_{1}^{\frac{R_{2}}{R_{1}}} \rho^{\prime} v_{z}^{\prime} r \mathrm{~d} r \mathrm{~d} \varphi=0,
$$

which ensures the absence of the fluid discharge through the cavity cross-section 
of the cylinders.

Let us assume that on the surface of the cylinders the following boundary conditions

$$
\begin{array}{ll}
v_{r}^{\prime}=U_{0}, & v_{\varphi}^{\prime}=0, \quad v_{z}^{\prime}=0, \quad T^{\prime}=T_{1} \quad\left(r=R_{1}\right), \\
v_{r}^{\prime}=\frac{U_{0}}{R}, & v_{\varphi}^{\prime}=0, \quad v_{z}^{\prime}=0, \quad T^{\prime}=T_{2} \quad\left(r=R_{2}\right),
\end{array}
$$

are fulfilled, where $R=\frac{R_{2}}{R_{1}}$.

Problem (1)-(3) possesses a group of symmetries $\mathbf{G}=S O(2) * O(2)$ which are invariant with respect to rotations $L_{\varphi}^{\delta}$ near the cylinder axis, to shifts $L_{\varphi}^{h}$ along that axis, and also to inversion $J$. These factors act on the vector-function $F\left(v_{r}, v_{\varphi}, v_{z}, T\right)$ according to the rules

$$
\begin{aligned}
& \left(L_{\varphi}^{\delta} F\right)(t, r, \varphi, z)=F(t, r, \varphi+\delta, z), \\
& \left(L_{z}^{h} F\right)(t, r, \varphi, z)=F(t, r, \varphi, z+h), \\
& (J F)(t, r, \varphi, z)=\left\{v_{r}(t, r, \varphi,-z), v_{\varphi}(t, r, \varphi,-z),-v_{z}(t, r, \varphi,-z), T(t, r, \varphi,-z)\right\}
\end{aligned}
$$

for any real $\delta$ and $h$.

As is easily seen, problem (1)-(3) admits an exact solution

$$
\begin{array}{ll}
V_{0}=\left\{u_{0}(r), v_{0}(r), 0\right\}, & T_{0}=c_{1}+c_{2} r^{\varkappa P_{r}}, \\
u_{0}(r)=\frac{R_{1} U_{0}}{r}, \quad v_{0}(r)= \begin{cases}\frac{K}{\varkappa}\left(a r^{\varkappa+1}+\frac{b}{r}-r\right), & \varkappa \neq-2, \\
\frac{K}{2}\left(\frac{a_{1} \ln r+b_{1}}{r}\right), & \varkappa=-2,\end{cases} \\
\frac{\partial \Pi_{0}}{\partial r}=\frac{\rho\left(u_{0}^{2}+v_{0}^{2}\right)}{r},
\end{array}
$$

where

$$
\begin{gathered}
K=\frac{1}{2 \rho \nu}\left(\frac{\partial \Pi_{0}}{\partial \theta}\right)_{0}, \quad a=\frac{R^{2}-1}{\left(R^{\varkappa+2}-1\right) R_{1}^{\varkappa}}, \quad a_{1}=\frac{R_{1}^{2}\left(R^{2}-1\right)}{\ln R}, \\
b=\frac{R_{2}^{2}\left(R^{\varkappa}-1\right)}{R^{\varkappa+2}-1}, \quad b_{1}=-\frac{R_{1}^{2} \ln R_{2}-R_{2}^{2} \ln R_{1}}{\ln R}, \\
c_{1}=\frac{T_{1} R^{\operatorname{Pr} \varkappa}-T_{2}}{R^{\varkappa \operatorname{Pr}}-1}, \quad c_{2}=\frac{T_{2}-T_{1}}{R_{1}^{\varkappa \operatorname{Pr}}-1}
\end{gathered}
$$

and $\varkappa=\frac{U_{0} R_{1}}{v}$ is the radial Reynolds number, $P_{r}=\frac{v}{\chi}$ is the Prandtl number.

The radial flow is inward for $\varkappa<0$ (converging flow) and outward for $\varkappa>0$ (diverging flow).

The flow with the velocity vector $V_{0}$, temperature $T_{0}$ and pressure $\Pi_{0}$ will be called the Dean stationary flow of a heat-conducting fluid with a radial temperature gradient and a radial flow. 


\section{Nonlinear System for Perturbations}

A solution of problem (1)-(3) is sought in the form

$$
V^{\prime}=V_{0}+V\left(v_{r}, v_{\varphi}, v_{z}\right), \quad T^{\prime}=T_{0}+T, \quad \Pi^{\prime}=\Pi_{0}+\Pi .
$$

Taking into account that the main stationary flow is a rotating shear flow, we denote rotation shear $S$ by $\frac{V_{m}}{d}$, where $V_{m}$ is an average velocity in the azimuthal direction, $d=R_{2}-R_{1}$ is a gap width between cylinders. Introducing dimensionless variables for time, velocity, temperature and pressure by $S, R_{2}, S R_{2}, T_{2}-T_{1}$, $v \rho^{\prime} S$ in the system (1), for the vector-functions $F=\left\{v_{r}, v_{\varphi}, v_{z}, T\right\}$ and $F_{1}=\left\{u_{r}, u_{\varphi}, u_{z}, T_{1}\right\}$, we obtain the following nonlinear problem of finding perturbations $V, T$ and $\Pi$ :

$$
\begin{aligned}
& \frac{\partial F}{\partial t}+N F-\frac{1}{\operatorname{Re}} M F+\frac{1}{\operatorname{Re}} \nabla_{1} \Pi=-\mathcal{L}(F, F), \\
& \left(\nabla_{1}, r F\right)=0,\left.\quad F\right|_{r=1, R}=0,
\end{aligned}
$$

where

$$
\begin{aligned}
& M F=\left\{\Delta_{1} v_{r}-\frac{1-\varkappa}{r^{2}} v_{r}-\frac{2}{r^{2}} \frac{\partial v_{\varphi}}{\partial \varphi}, \Delta_{1} v_{\varphi}-\frac{1+\varkappa}{r^{2}} v_{\varphi}+\frac{2}{r^{2}} \frac{\partial v_{r}}{\partial \varphi}, \Delta_{1} v_{z}, \frac{1}{\operatorname{Pr}} \Delta_{1} T\right\}, \\
& N F=\omega_{1} \frac{\partial F}{\partial \varphi}+\left\{\operatorname{Ra} \omega_{2} T-2 \omega_{1} v_{\varphi},-g_{1} v_{r}, 0, \frac{g_{2}}{\operatorname{Pr}} v_{r}\right\}, \\
& \mathcal{L}\left(F, F_{1}\right)=\left\{\left(F, \nabla_{1}\right) u_{r}-\frac{v_{\varphi} u_{\varphi}}{r},\left(F, \nabla_{1}\right) u_{\varphi}+\frac{v_{r} u_{\varphi}}{r},\left(F, \nabla_{1}\right) u_{z},\left(F, \nabla_{1}\right) T_{1}\right\} \text {, } \\
& \Delta_{1}=\frac{\partial^{2}}{\partial r^{2}}+\frac{1-\varkappa}{r} \frac{\partial}{\partial r}+\frac{1}{r^{2}} \frac{\partial^{2}}{\partial \varphi^{2}}+\frac{\partial^{2}}{\partial z^{2}}, \quad \nabla_{1}=\left\{\frac{\partial}{\partial r}, \frac{1}{r} \frac{\partial}{\partial \varphi}, \frac{\partial}{\partial z}, 0\right\}, \\
& \operatorname{Re}=\frac{V_{m} d}{v} \text { is the azimuthal Reynolds number, } \\
& \mathrm{Ra}=\frac{\beta\left(T_{2}-T_{1}\right)}{2} \text { is the Rayleigh number, } \\
& \omega_{1}=\frac{V_{m} g(r)}{r}, \quad \omega_{2}=\omega_{1}^{2} r, \quad V_{m}=\frac{K}{\varkappa} \cdot \frac{d}{R_{2}} D 4, \\
& g(r)=\frac{d}{R_{2}} \frac{D 1 r^{\varkappa+2}+D 2-r^{2}}{r D 4}, \\
& g_{1}(r)=\frac{\mathrm{d} v_{0}}{\mathrm{~d} r}+\frac{v_{0}}{r}=\frac{K}{\varkappa} \cdot \frac{d}{R_{2}} \frac{D 1(\varkappa+2) r^{\varkappa}-2}{D 4} \text {, } \\
& g_{2}(r)=\frac{\varkappa \operatorname{Pr}^{2} \cdot R^{\varkappa \operatorname{Pr}}}{R^{\varkappa \operatorname{Pr}}-1} r^{\varkappa \operatorname{Pr}}-1, \\
& D 1=\frac{\left(R^{2}-1\right) R^{\varkappa}}{R^{\varkappa+2}-1}, \quad D 2=1-D 1, \\
& D 3=\frac{R^{2}-1}{R^{2}}, \quad D 4=D 2 \ln R-\frac{\varkappa D 3}{2(\varkappa+2)} .
\end{aligned}
$$


Note, that $D_{e}=\operatorname{Re}\left(\frac{d}{R_{2}}\right)^{\frac{1}{2}}$ is the Dean number.

Problem (7) is written in terms of the Boussinesq approximation, which is based on the assumption that the thermal expansion coefficient is small [27]. In the sequel it will always be assumed that the velocity, temperature and pressure components are periodic with respect to $z$ and $\varphi$ with the known periods $2 \pi / \alpha$ and $2 \pi / m$, respectively. Thus the solution of the problem depends on the 7 dimensional parameters: azimuthal and radial Reynolds numbers, Rayleigh and Prandtl numbers, the ratio of the radii of cylinders $R$, axial and azimuthal wave numbers $\alpha, m$, respectively.

\section{Neutral Curves}

The theoretical and experimental studies have shown that after the loss of stability of Dean flow there occured secondary modes via either axisymmetric or nonaxisymmetric disturbances as Dean vortices and oscillatory modes in the form of traveling waves. To study the transition to complex regimes of special attention are the points of intersection of neutral curves, corresponding to the two above-mentioned kinds of the secondary flows, since at these points with a high probability may appear various regimes, including the complex one.

Let $\left(\operatorname{Ra}_{0}, \mathrm{Re}_{0}\right)$ be the point lying on the plane of parameters $(\mathrm{Ra}, \mathrm{Re})$ and corresponding to the intersection of the neutral curves corresponding to the monotonic $(m=0)$ axisymmetric and oscillatory nonaxisymmetric loss of stability of main flow (3). In this paper we will explore the complex modes that may appear in the vicinity of intersection points of neutral curves corresponding to the vortex and azimuthal wave bifurcations. Under the definite values of parameters of the problem, the neutral curves may be nonintersecting that indicates that under the corresponding values of parameters of the problem we cannot expect the appearance of complex regimes.

To construct neutral curves, we assume that the perturbations $V$, temperature $T$ and pressure $\Pi$ are infinitely small. Neglecting in (7) the nonlinear terms and seeking for a solution of that problem in the case of axisymmetric monotone perturbations, we obtain the following spectral problem

$$
(M-\operatorname{Re} N) \Phi_{0}=\nabla_{1} p_{0}, \quad\left(\nabla_{1}, r \Phi_{0}\right)=0,\left.\quad \Phi_{0}\right|_{r=1, R}=0,
$$

where

$$
\Phi_{0}=\left\{u_{0}(r), v_{0}(r), i w_{0}(r), \tau_{0}(r)\right\} \mathrm{e}^{i \alpha z}, \quad p_{0}=q_{0}(r) \mathrm{e}^{i \alpha z} .
$$

By the substitution of (9) into (8), after dividing the variables in (8) we obtain the spectral problem for the ordinary differential equations

$$
\begin{aligned}
& \left(L-\frac{1-\varkappa}{r^{2}}-\alpha^{2}\right) u_{0}=\frac{\mathrm{d} q_{0}}{\mathrm{~d} r}-2 \operatorname{Re} \omega_{1} v_{0}+\operatorname{Re} \operatorname{Ra} \omega_{2} \tau_{0}, \\
& \left(L-\frac{1+\varkappa}{r^{2}}-\alpha^{2}\right) v_{0}=-\operatorname{Re} g_{1} u_{0},
\end{aligned}
$$




$$
\begin{aligned}
& \left(L-\alpha^{2}\right) w_{0}=\alpha q_{0}, \quad\left(L-\alpha^{2}\right) \tau_{0}=\operatorname{Re} g_{2} u_{0}, \quad \frac{\mathrm{d} u_{0}}{\mathrm{~d} r}+\frac{u_{0}}{r}-\alpha w_{0}=0, \\
& u_{0}=v_{0}=w_{0}=\tau_{0}=0 \quad(r=1, R) \text {, } \\
& \text { where } L=\frac{\mathrm{d}^{2}}{\mathrm{~d} r^{2}}+\frac{1-\varkappa}{r} \frac{\mathrm{d}}{\mathrm{d} r} \text {. }
\end{aligned}
$$

The neutral curves corresponding to the bifurcation of azimuthal waves origination are found by solving the spectral problem

$$
(M-\operatorname{Re} N-i c \operatorname{Re}) \Phi_{1}=\nabla_{1} p_{1}, \quad\left(\nabla_{1}, r \Phi_{1}\right)=0,\left.\quad \Phi_{1}\right|_{r=1, R}=0 .
$$

A solution of problem (11) is sought in the form

$$
\Phi_{1}=\left\{u_{1}(r), v_{1}(r), w_{1}(r), \tau_{1}(r)\right\} \mathrm{e}^{-i(m \varphi+\alpha z)}, \quad p_{1}=q_{1}(r) \mathrm{e}^{-i(m \varphi+\alpha z)},
$$

where $c$ is the unknown frequency of autooscillations, $m$ is the azimuthal wave number. Substitution of (12) into (11) after dividing the variables in (11), we get the spectral problem for the ordinary differential equations:

$$
\begin{aligned}
& {\left[L_{1}-\frac{1-\varkappa}{r^{2}}-\alpha^{2}-i \operatorname{Re}\left(c-m \omega_{1}\right)\right] u_{1}=\frac{\mathrm{d} q_{1}}{\mathrm{~d} r}-2 \operatorname{Re} \omega_{1} v_{1}+\operatorname{Re} \operatorname{Ra} \omega_{2} \tau_{1}-\frac{2 i m}{r^{2}} v_{1},} \\
& {\left[L_{1}-\frac{1+\varkappa}{r^{2}}-\alpha^{2}-i \operatorname{Re}\left(c-m \omega_{1}\right)\right] v_{1}=-\frac{i m}{r} q_{1}-\operatorname{Re} g_{1}(r) u_{1}+\frac{2 i m}{r^{2}} u_{1},} \\
& {\left[L_{1}-\alpha^{2}-i \operatorname{Re}\left(c-m \omega_{1}\right)\right] w_{1}=-i \alpha q_{1},} \\
& {\left[L_{1}-\alpha^{2}-i \operatorname{Re} \operatorname{Pr}\left(c-m \omega_{1}\right)\right] \tau_{1}=\operatorname{Re} g_{2} u_{1},} \\
& \frac{\mathrm{d} u_{1}}{\mathrm{~d} r}+\frac{u_{1}}{r}-\frac{i m}{r} v-i \alpha w_{1}=0, u_{1}=v_{1}=w_{1}=\tau_{1}=0 \quad(r=1, R),
\end{aligned}
$$

where

$$
L_{1}=\frac{\mathrm{d}^{2}}{\mathrm{~d} r^{2}}+\frac{1-\varkappa}{r} \frac{\mathrm{d}}{\mathrm{d} r}-\frac{m^{2}}{r^{2}} .
$$

Problems of eigenvalues (10) and (13) have been solved by the shooting method for fixed $\varkappa, \alpha, R, m, \operatorname{Pr}, \mathrm{Ra}$. Thus, for the fixed values of these parameters we established the dependence of the critical value of the azimuthal Reynolds number Re and the neutral mode frequency $c$ corresponding to the bifurcation of vortices and azimuthal waves origination on a Rayleigh number Ra. Further, using the Newton method, we minimize the difference between the obtained critical values of Re. This allows us to calculate with sufficient exactness the values $\mathrm{Ra}_{0}, \mathrm{Re}_{0}$ and $c_{0}$ corresponding to the point of intersection of neutral curves.

The calculations in this paper were performed for the case $R=2$ (radius of the outer cylinders is two times greater than that of the inner ones), $m=0,1$, for various values of axial wave number $\alpha, \operatorname{Pr}=0.71$ and for small absolute values $\varkappa(3 \leq \varkappa \leq 3)$. The results of calculations are presented in Table 1.

As our calculations show, these intersections of neutral curves take place especially when temperature of the outer cylinder is higher than that of the inner one $\left(\mathrm{Ra}_{0}>0\right)$ for sufficiently large values of the wave axial number and when 
Table 1. The points of intersection of neutral curves.

\begin{tabular}{cccccccccc}
\hline & \multicolumn{3}{c}{$\alpha=8$} & \multicolumn{3}{c}{$\alpha=5$} & \multicolumn{3}{c}{$\alpha=4$} \\
\cline { 2 - 9 } & $\mathrm{Ra}_{0}$ & $\mathrm{Re}_{0}$ & $c_{0}$ & $\mathrm{Ra}_{0}$ & $\mathrm{Re}_{0}$ & $c_{0}$ & $\mathrm{Ra}_{0}$ & $\mathrm{Re}_{0}$ & $c_{0}$ \\
\hline 3 & 3.627 & 20.9 & 2.795 & 0.714 & 68.445 & 2.473 & 0.873 & 87.84 & 2.49 \\
2 & 3.14 & 22.07 & 2.792 & 0.667 & 82.88 & 2.458 & 0.809 & 81.245 & 2.472 \\
1.5 & 2.52 & 24.45 & 2.7826 & 0.65 & 59.86 & 2.456 & 0.778 & 77.421 & 2.4709 \\
0.5 & 2.084 & 26.938 & 2.763 & 0.64 & 53.09 & 2.473 & 0.75 & 68.464 & 2.492 \\
0.2 & 1.675 & 30.2 & 2.727 & 0.65 & 51.488 & 2.48 & 0.7524 & 65.438 & 2.507 \\
-0.3 & - & - & - & 0.7 & 46.25 & 2.52 & 0.772 & 60.164 & 2.541 \\
-0.5 & - & - & - & 0.72 & 44.73 & 2.525 & 0.785 & 58.028 & 2.558 \\
-1 & - & - & - & 0.84 & 39.73 & 2.593 & 0.835 & 52.86 & 2.606 \\
-1.5 & - & - & - & 1.036 & 34.038 & 2.6678 & 0.902 & 48.268 & 2.6604 \\
-3 & - & - & - & 1.784 & 25.479 & 2.877 & 1.157 & 39.24 & 2.824 \\
\hline
\end{tabular}

the liquid moves through the inner cylinder to the outer one. This indicates that short-wave axially directed perturbations generating vortices and azimuthal waves are interacting, and hence one may expect the appearance of complex regimes. If values of the axial wave numbers decrease, i.e., for the corresponding long-wave perturbations there are also an intersections of neutral curves for the converging flow.

If temperature of the inner cylinder exceeds that of the outer one $\left(\operatorname{Ra}_{0}<0\right)$, neutral curves do not intersect, and thus it is difficult to expect the occurrence of complex modes.

\section{The Amplitude System and Transitions}

To investigate the secondary flows and the appearance of high instabilities in the flow (3) we use the nonlinear theory of bifurcation of hydrodynamic flows with cylindrical symmetry (see [28]-[33]).

This theory allows one to find various liquid motion regimes existing in the vicinity of the points of intersection of neutral curves corresponding to the two types of secondary instability-vortices and azimuthal waves in the Dean flow. This theory has been applied in a rather wide class of problems, such, for example, as the Couette flow [34], the flow between permeable cylinders [35] [36] [37] [38] [39], a heat-conducting liquid flow between rigid and permeable cylinders [40] [41] [42] [43] [44].

The problems are reduced to the investigation of nonlinear dynamical autonomous systems of the amplitude equation, which is a generalization of Landaus amplitude equation.

The $S O(2) * O(2)$ symmetry enables one to reduce the six-dimensional amplitude system with unknown complex amplitudes $\eta_{0}, \eta, \eta_{1}$ to the four-dimensional motor subsystem for the modules of the amplitudes with free parameters $\sigma, \mu$ (the damping decrements of the monotonic and oscillatory 
perturbations, respectively). To the equilibria of this system, which is lying on the invariant subspaces, there correspond the motions of a fluid, which has the concrete physical nature [32]-[44]: main stationary flow; vortex flows, i.e., a secondary stationary axisymmetric flow; purely azimuthal waves, i.e., secondary oscillatory modes; spiral waves, i.e., secondary autooscillatory modes; mixed azimuthal waves, i.e., three frequency regimes; equilibria not lying on the invariant subspaces, i.e., equilibria of a general state, each of them corresponds to a quasiperiodic two-frequence solution of the amplitude system.

As our calculations shows, depending on the parameter values of the problem, the motor subsystem may have no equilibria or may have equilibria of the above mentioned types. It is found, that transition schemes may turn out to be both rather complicated and absolute trivial.

We present here the scheme of equilibria bifurcations of the motor subsystem, which we consider most interesting and allowing us to judge about the transitions characteristic of the considered system of small absolute values of radial Reynolds number $\varkappa$.

Consider in detail the case for $\varkappa=3$ (dividing flow), $\alpha=4$ (perturbations are $\frac{\pi}{2}$ periodical in the axial direction), $\mathrm{Ra}_{0}=0.873$ (temperature of the outer cylinder is higher than that of the inner one).

For $\sigma<0$ and $\mu_{r}<0 \quad\left(\mu_{r}\right.$ is the real part of damping decrement of oscillatory perturbations $\mu$ ) the equilibria fail to take place, but for $\mu_{r}>0$ of all the equilibria of the motor subsystem there exist only unstable main stationary flow and unstable purely azimuthal waves.

For $\sigma=0$ and $\mu_{r}<0$ there exist only stable main stationary flow,but for $\mu_{r}>0$ there are unstable main stationary flow and vortices.

For $\sigma>0$ and $\mu_{r}<0$ the unstable main stationary flow and vortices exists fon any values of free parameters; vortices are stable for $\mu_{r}<\mu_{r}^{2}$ and unstable for $\mu_{r}>\mu_{r}^{2}$.

For $\sigma>0, \mu_{r}>0$ the transitions associated with bifurcations are graphically shown in Figure 2.

The single lines show $J$-symmetric equilibria, the double lines indicate $J$-connected pair of equilibria. Stable equilibria are drawn by solid lines and unstable equilibria by dotted lines. The circles are the points at which the motor subsystem cycles bifurcate.

For $\mu_{r}=0$ from main flow (3) there simultaneowly bifurcate unstable pure azimuthal waves and unstable $J$-connected pair of spiral waves. Spiral waves are stable for $\mu_{r}>\mu_{r}^{4}$, for $\mu_{r}=\mu_{r}^{4}$ from them bifurcates an unstable connected pair of general equilibria. This pair exists in the range $\mu_{r}^{4}<\mu_{r}<\mu_{r}^{8}$ and disappear for $\mu_{r}=\mu_{r}^{8}$ merging with unstable mixed azimuthal waves. On the other hand at the point $\mu_{r}=\mu_{r}^{9}$ mixed azimuthal waves merging with purely azimuthal waves.

For $\mu_{r}=\mu_{r}^{3}$, of a set of limit symmetric cycles of the motor subsystem in the form of number eight are branching off from mixed azimuthal waves and exists 


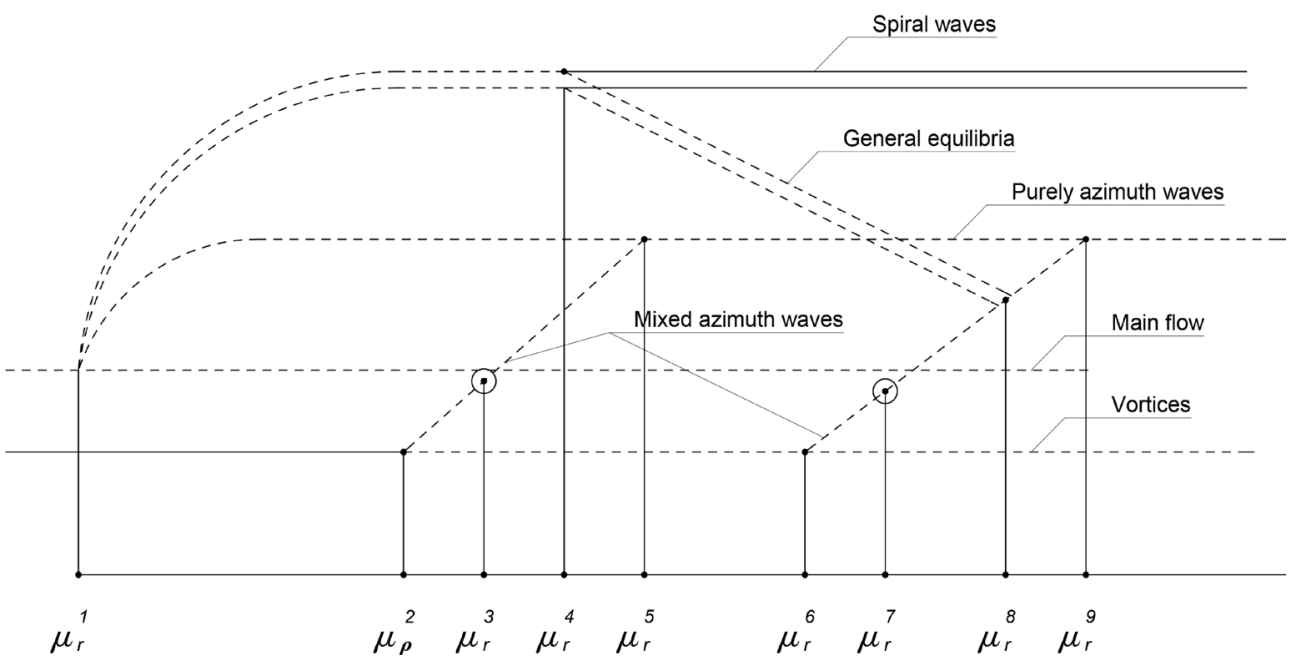

Figure 2. Bifurcation values: $\mu_{r}^{1}=0, \mu_{r}^{2}=2.75, \mu_{r}^{3}=2.95, \mu_{r}^{4}=3.025, \mu_{r}^{5}=3.265, \mu_{r}^{6}=3.8$, $\mu_{r}^{7}=4.003, \mu_{r}^{8}=4.05, \mu_{r}^{9}=4.1$.

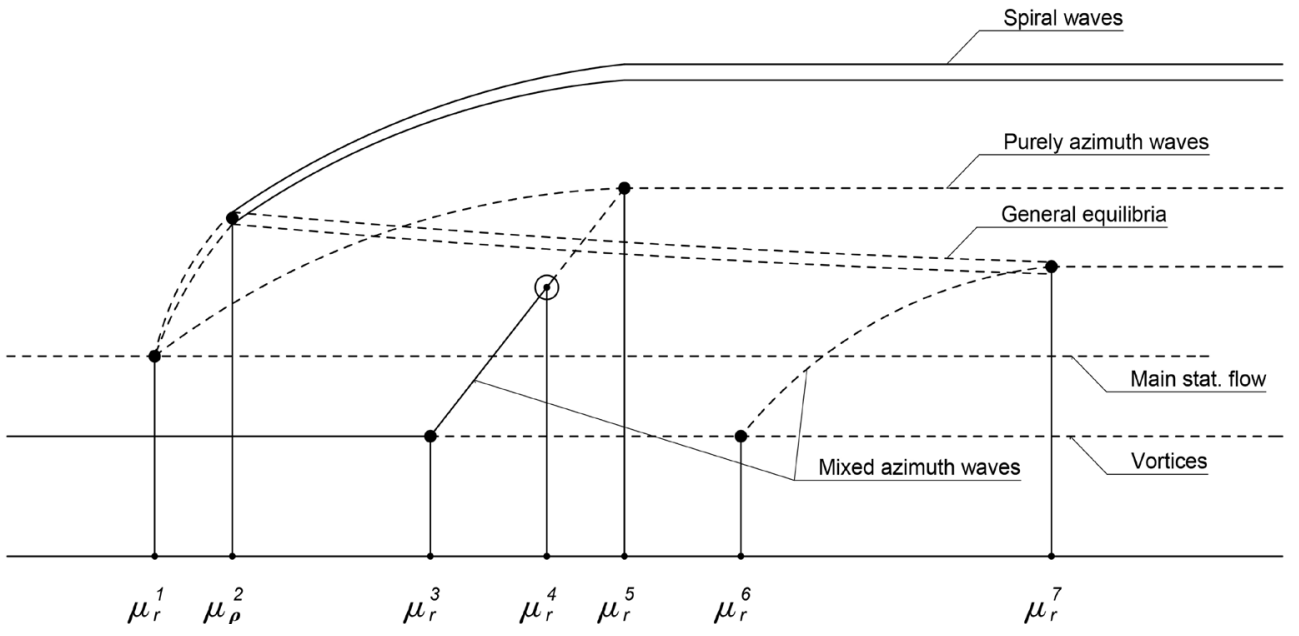

Figure 3. Bifurcation values: $\mu_{r}^{1}=0, \mu_{r}^{2}=0.8, \mu_{r}^{3}=4.259, \mu_{r}^{4}=4.88, \mu_{r}^{5}=6.3, \mu_{r}^{6}=10.009$, $\mu_{r}^{7}=15.285$.

in the range $\mu_{r}^{3}<\mu_{r}<\mu_{r}^{5}$. They were calculated by using the software prepared in [34] [42], which enabled us to investigate the evolution, stability and bifurcatios of cycles for various values of the problem. The limit cycles of similar forms as the phase trajectories branching off from the mixed azimuthal waves are repeatedly met in the works (see, for example, [34]-[44]) when considering concrete problems between rotating vertical cylinders. In our case, the cycles are unstable and have no bifurcations. They exists in the small range $4.003<\mu_{r}<4.01$ and merges with the mixed azimuthal waves.

Consider the case $\varkappa=-0.5$ (converging flow), $\alpha=4$ (perturbations are $\pi / 2$ periodical in the axial direction), the Rayleigh number $\mathrm{Ra}_{0}=0.785$ (temperature of the outer cylinder is higher than that of the inner) for $\sigma>0$. The transitions associated with the bifurcation are graphically shown in Figure 3. 
In comparison to the case under consideration for diverging flow, the picture of bifurcations for converging flow becomes more complicated.

A characteristic feature of the transitions in this case are the existence of several stable equilibria in the range changes $\mu_{r}$, for example, $\mu_{r}^{2}<\mu_{r}<\mu_{r}^{4}$. For the values of a free parameter belonging to such ranges, in the experiments, the hysteresis phenomena are observed [42].

In this case, in contrast to the case $\varkappa>0$ for $\mu_{r}=\mu_{r}^{4}$, from the mixed azimuthal waves branch off the nonsymmetric limit stable cycles similar to the deformed number eight, they lose their stability because of bifurcations of the period duplications at the point $\mu_{r}=5.3$ and from it branches off a $J$-connected pair of stable II-revolution nonsymmetric cycles which exist for $5.3<\mu_{r}<5.7$. A further increase of $\mu_{r}$ leads to the formation of another series of duplications of $J$-connected pairs of cycles which for $\mu_{r}=5.9$ results in to the generation of $J$-connected pair of chaotic attractors; the form of their phase trajectories differs slightly from that of the trajectories of the generated cycles; changes gradually its form and disappears for $\mu_{r}=6.2$. It can be calculated in a very narrow range of variation of the parameter.In the same way, as well as with vertical cylinders (see [44]), we can judge about the appearance of complex regimes.

\section{Conclusions}

In paper under consideration, we have examined possible transitions to complex modes in a heat-conducting flow produced by pumping a fluid around the annulus of a system of fixed porous horizontal cylinders. The liquid is under the action of a radial flow and a radial temperature gradient.

1) Neutral curves corresponding to vortices and azimuthal waves are calculated.

2) It is established that for diverging flow with small absolute values of radial Reynolds numbers and for sufficiently large values of the wave axial number, the intersections of neutral curves take place especially when temperature of the outer cylinder is higher than that of the inner one $\left(\mathrm{Ra}_{0}>0\right)$. This indicates that the short-wave axially directed perturbations generating vortices and azimuthal waves are interacting, and hence one may expect the appearance of complex regimes. If values of the axial wave numbers decrease, i.e., for the corresponding long-wave perturbations there are also intersections of neutral curves for the converging flow.

If temperature of the inner cylinder exceeds that of the outer one $\left(\operatorname{Ra}_{0}<0\right)$, neutral curves do not intersect, and thus it is difficult to expect the occurrence of complex modes.

1) Equilibria of a motor subsystem which are lying on the invariant subspaces are calculated. They correspond to stationary or periodic regimes of the fluid motion.

2) Equilibria of a general state are calculated. They corresponds to a quasiperiodic 
two-frequency solution of the amplitude system.

3) It is shown that for a converging flow there are several stable equilibria on the range of change $\mu_{r}$. For the values of a free parameter belonging to such ranges, in the experiments, hysteresis phenomena are observed [42].

4) It is established that for the certain values of the problem, there exist stable or unstable limit cycles of the motor subsystem.

5) It is shown that for converging flow, when temperature of the outer cylinder is higher than that of the inner one, the sequential duplications of a period of stable limit cycles lead to the generation of chaotic attractors and consequently as well as with vertical cylinders [43], we can judge about the appearance of complex regimes.

\section{References}

[1] Eustice, J. (1910) Flow of Water in Curved Pipes. Proceedings of the Royal Society London, Series A, 84, 107-118. https://doi.org/10.1098/rspa.1910.0061

[2] Eustice, J. (1911) Experiments of Streamline Motion in Curved Pipe. Proceedings of the Royal Society London, Series A, 85, 119-131.

https://doi.org/10.1098/rspa.1911.0026

[3] Dean, W.R. (1928) The Stream-Line Motion of Fluid in a Curved Pipe. Philosophical Magazine, 30, 673-693. https://doi.org/10.1080/14786440408564513

[4] Reid, W.H. (1958) On the Stability of Viscous Flow in a Curved Channel. Proceedings of the Royal Society London, Series A, 244, 186-198. https://doi.org/10.1098/rspa.1958.0035

[5] Hämmerlin, G. (1957) Die Stabilität der Strmung in einem gekrümmten Kanal, Arch. Archive for Rational Mechanics and Analysis, 1, 212-224. (In Germany) https://doi.org/10.1007/BF00298005

[6] Brewster, D.B., Grosberg P. and Nissan, A.H. (1959) The Stability of Viscous Flow Between Horizontal Concentric Cylinders. Proceedings of the Royal Society London, Series A, Mathematical, Physical and Engineering Sciences, 251, 76-91. https://doi.org/10.1098/rspa.1959.0091

[7] Chandrasekhar S. (1961) Hydrodynamic and Hydromagnetic Stability. Clarendon Press, Oxford, 652 pp.

[8] Gibson, R.D. and Cook, A.E. (1974) The Stability of Curved Channel Flow. The Quarterly Journal of Mechanics and Applied Mathematics, 27, 140-160. https://doi.org/10.1093/qjmam/27.2.149

[9] Ligrani, P.M. and Niver, R.D. (1988) Flow Visualization of Dean Vortices in a Curved Channel with 40 to 1 Aspect Ratio. Physics of Fluids, 31, 3605-3617. https://doi.org/10.1063/1.866877

[10] Gelfgat, A.Yu. and Yarin, A.L. and Bar-Yoseph, P.Z. (2001) Three-Dimensional Instability of a Two-Layer Dean Flow. Physics of Fluids, 13, 3185-3195. https://doi.org/10.1063/1.1409967

[11] Le Cunff, C. and Bottaro, A. (1993) Linear Stability of Shear Profiles and Relation to the Secondary Instability of the Dean Flow. Physics of Fluids, 5, 2161-2171. https://doi.org/10.1063/1.858555

[12] Mees, P.A.J., Nandakumar, K. and Masliyah, J.H. (1996) Secondary Instability of Flow in a Curved Duct of Square Cross-Section. Journal of Fluid Mechanics, 323, 387-409. https://doi.org/10.1017/S0022112096000973 
[13] Matsson, O.J.E. and Alfredsson, P.H. (1990) Curvature and Rotation-Induced Instabilities in Channel Flow. Journal of Fluid Mechanics, 210, 537-563. https://doi.org/10.1017/S0022112090001392

[14] Moore, J.E., Xu, C., Glagov, S., Zarins, C.K. and Ku, D.N. (1994) Fluid Wall Shear Stress Measurements in a Model of the Human Abdominal Aorta: Oscillatory Behavior and Relationship to Atherosclerosis. Atherosclerosis, 110, 225-240.

[15] Shahcheraghi, N., Dwyer, H.A., Cheer, A.Y., Barakat, A.I. and Rutaganira, T. (2002) Unsteady and Three-Dimensional Simulation of Blood Flow in the Human Aortic Arch. Journal of Biomechanical Engineering, 124, 378-387. https://doi.org/10.1115/1.1487357

[16] Mohammad, M.H., Mohammad, M.A., Mohammad, F. and Osman, A.B. (2013) Numerical Simulation of Dean Number and Curvature Effects on Magneto-Biofluid Flow through a Curved Conduit. Proceedings of the Institution of Mechanical Engineers, Part H, 227, 1155-1170.

[17] Issacci, F., Ghoniem, N.M. and Catton, I. (1988) Magnetohydrodynamic Flow in a Curved Pipe. Physics of Fluids, 31, 65-71. https://doi.org/10.1063/1.866578

[18] Deka, R.K. and Takhar, H.S. (2004) Hydrodynamic Stability of Viscous Flow between Curved Porous Channel with Radial Flow. International Journal of Engineering Science, 42, 953-966.

[19] Ali, M.A., Takhar, H.S. and Soundalgekar, V.M. (1998) Effect of Radial Temperature Gradient on the Stability of Flow in a Curved Channel. Proceedings of the Royal Society of London, Mathematical, Physical and Engineering Science, 454, 2279-2287. https://doi.org/10.1098/rspa.1998.0259

[20] Deka, R.K. and Paul, A. (2013) Stability of Dean Flow between Two Porous Concentric Cylinders with Radial Flow and a Constant Heat Flux at the Inner Cylinder. Journal of Fluids Engineering, 135, Article ID: 041203. https://doi.org/10.1115/1.4023661

[21] Deka, R.K., Gupta, A.S. and Das, S.K. (2007) Stability of Viscous Flow Driven by an Azimuthal Pressure Gradient between Two Porous Concentric Cylinders with Radial Flow and a Radial Temperature Gradient. Acta Mechanica, 189, 73-86. https://doi.org/10.1007/s00707-006-0399-3

[22] Finlay, W.H., Keller, J.B. and Ferziger, J.H. (1988) Instability and Transition in Curved Channel Flow. Journal of Fluid Mechanics, 194, 417-456. https://doi.org/10.1017/S0022112088003052

[23] Aider, A.A. and Kadem, L. (2011) Transition to Turbulence of the Dean and Taylor-Couette Flows: Similarities, Multiphysics, Barselona. http://www.ummto.dz/IMG/pdf/Multiphysics_Barcelona_2011.pdf

[24] Matsson, O.J.E. and Alfredsson, P.H. (1993) Secondary Instability and Breakdown to Turbulence in Curved Channel Flow. Applied Scientific, Research, 51, 9-14.

[25] Bland, S.B. and Finlay, W.H. (1991) Transitions towards Turbulence in a Curved Channel. Physics of Fluids, 3, 106-114. https://doi.org/10.1063/1.857870

[26] Landau, L.D. and Lifshits, E.M. (1987) Fluid Mechanics. Vol. 6, 2nd Edition, Butterworth-Heinemann.

[27] Gershuni, G.Z. and Zhukhovitski, E.M. (1976) Convective Stability of Incompressible Fluid. Keter, Jerusalem/Wiley.

[28] Yudovich, V.I. (1966) The Bifurcation of a Rotating Flow of Liquid. Soviet Physics, Doklady, 11, 566.

[29] Yudovich, V.I. (1966) Secondary Flows and Fluid Instability between Rotating Cy- 
linders. Journal of Applied Mathematics and Mechanics, 30, 822-833.

[30] Yudovich, V.I. (1967) Stability of Convection Flows. Journal of Applied Mathematics and Mechanics, 31, 294-303.

[31] Iooss, G. (1984) Bifurcation and Transition to Turbulence in Hydrodynamics. In: Salvadori, L., Ed., Bifurcation Theory and Applications, Lecture Notes in Mathematics, Vol. 1057, Springer, Berlin, Heidelberg. https://doi.org/10.1007/BFb0098596

[32] Chossat, P. and Iooss, G. (1985) Primary and Secondary Bifurcations in the Couette-Taylor Problem. Japan Journal of Industrial and Applied Mathematics, 2 , 37-68.

[33] Chossat, P. and Iooss, G. (1994) The Couette-Taylor Problem. Springer-Verlag, New York. https://www.books.google.com

[34] Kolesov, V.V. and Yudovich, V.I. (1998) Calculation of Oscillatory Regimes in Couette ow in the Neighborhood of the Point of Intersection of Bifurcations Initiating Taylor Vortices and Azimuthal Waves. Fluid Dynamics, 33, 532. https://doi.org/10.1007/BF02698218

[35] Kolesov, V.V. and Shapakidze, L.D. (2000) On Transitions near the Intersection Point of Bifurcations in the ow between Two Rotating Permeable Cylinders. Proceedengs of $A$. Razmadze Mathematical Institute, 122, 79-91. http://rmi.tsu.ge/proceedings/

[36] Kolesov, V.V. and Shapakidze, L.D. (1999) On Oscillatory Modes in Viscous Incompressible Liquid Flows between Two Counter-Rotating Permeable Cylinders. In: Iooss, G., Gues, O. and Nouri, A., Eds., Trends in Applications of Mathematics to Mechanics, Vol. 106, CRC Press, Boca Raton, 221-227. https://books.google.com

[37] Kolesov, V.V. and Romanov, M.N. (2010) Calculation of the Stationary, Periodic, and Quasi-Periodic Viscous Flows between Two Rotating Permeable Cylinders. Fluid Dynamics, 45, 880-888. https://doi.org/10.1134/S0015462810060050

[38] Kolesov, V.V. and Romanov, M.N. (2014) Occurrence of Quasiperiodic Flows between Two Rotating Permeable Cylinders. Journal of Applied Mechanics and Technical Physics, 55, 421-429. https://doi.org/10.1134/S0021894414030067

[39] Kolesov, V.V. and Romanov, M.N. (2013) Chaos Generation in the Couette-Taylor Problem for Permeable Cylinders. Fluid Dynamics, 48, 46-56. https://doi.org/10.1134/S0015462813010067

[40] Kolesov, V.V. (1981) Calculation of Auto-Oscillations Resulting from the Loss of Stability of a Nonisotherma Couette Flow. Fluid Dynamics, 16, 344-350. https://doi.org/10.1007/BF01092365

[41] Kolesov, V.V. (1981) Occurrence of a Two-Dimensional Secondary Regime between Heated Rotating Cylinders. Fluid Dynamics, 16, 817-822. https://doi.org/10.1007/BF01089707

[42] Kolesov, V.V. and Khoperski, A.G. (2009) Nonisothermal Couette-Taylor Problem. Yuj. Fed. Yniv. Rostov. (In Russian)

[43] Kolesov, V.V. and Khoperski, A.G. (2002) Simple Regimes of Uid Motion in the Neighborhood of the Intersection of Bifurcations Initiating Nonisothermal Taylor Vortices and Azimuthal Waves. Fluid Dynamics, 37, 257-267. https://doi.org/10.1023/A:1015862417312

[44] Kolesov, V.V. and Shapakidze, L.D. (2011) Instabilities and Transition in Flows between Two Porous Concentric Cylinders with Radial Flow and Radial Temperature Gradient. Physics of Fluids, 23, Article ID: 014107. https://doi.org/10.1063/1.3534026 
Submit or recommend next manuscript to SCIRP and we will provide best service for you:

Accepting pre-submission inquiries through Email, Facebook, LinkedIn, Twitter, etc. A wide selection of journals (inclusive of 9 subjects, more than 200 journals)

Providing 24-hour high-quality service

User-friendly online submission system

Fair and swift peer-review system

Efficient typesetting and proofreading procedure

Display of the result of downloads and visits, as well as the number of cited articles Maximum dissemination of your research work

Submit your manuscript at: http://papersubmission.scirp.org/

Or contact jamp@scirp.org 